\title{
Inhibitors of the renin-angiotensin system in established cardiac failure
}

\author{
Kanu Chatterjee
}

Congestive heart failure is a common clinical syndrome and recent epidemiologic surveys indicate that its incidence is increasing. It has been estimated that as many as four million people may suffer from heart failure in the United States. ${ }^{1}$ In the United Kingdom, the estimated prevalence is 3.9 per 1000 patients, and worldwide over 15 million patients are thought to have heart failure. ${ }^{2}$ The rate of hospital admission for heart failure, which imposes a considerable burden on health care costs, is also increasing in recent years and it is estimated that there are almost 650000 admissions per year for management of the condition in the United States. The incidence of heart failure increases with increasing age, especially over 65-70 years. In addition, hospital admissions are also frequent in those over 65 years old as compared to those aged 45-64, and more common in females than males.

Although congestive heart failure is a common clinical syndrome, the overall prognosis-once heart failure develops and regardless of the underlying disease-remains uniformly poor. Early reports have indicated that from the time of diagnosis, only half of all patients with established heart failure survive five years. It has also been observed that the more severe the heart failure is clinically, the worse the prognosis. In patients with New York Heart Association (NYHA) functional class IV, the overall survival at one year was between $34 \%$ and $52 \%{ }^{3}$ Recent epidemiological studies also suggest that there has been little improvement in the prognosis over the years, despite dramatic advances in the understanding of the pathophysiology of heart failure. In patients with coronary artery disease who developed congestive heart failure after acute myocardial infarction, five year mortality was $65 \%$ for men and $47 \%$ for women. These mortality rates are not significantly different from those reported several years earlier. ${ }^{3}$ Thus the ideal objectives for treatment of heart failure are: (1) to relieve symptoms and improve quality of life; (2) to improve prognosis; and (3) to reduce the health care cost, particularly by reducing the rate of hospital admissions for

\section{Glossary of trials}

AIRE-Acute Infarction Ramipril Efficacy study

CONSENSUS I-Cooperative New Scandinavian Enalapril Survival Study

SOLVD-Studies of Left Ventricular Dysfunction treatment of heart failure. During the past two decades, it has been shown that modulation of the renin-angiotensin system can provide substantial benefits in patients with heart failure in relieving symptoms and improving prognosis. Thus the major goals of treatment of heart failure can at least partially be achieved through this mechanism.

\section{Rationale for modulation of the renin- angiotensin system in established heart failure}

The activation of the renin-angiotensin-aldosterone axis in overt heart failure has been amply demonstrated in numerous clinical studies. $^{4-6}$ As early as 1946, Merrill et al reported that renin levels were increased in patients with congestive heart failure, ${ }^{7}$ concentrations of renal vein renin being significantly increased compared to normal subjects. Subsequently, many studies have reported that plasma renin activity is substantially increased both in experimental models of heart failure and in patients with overt clinical heart failure. ${ }^{5}$ As expected, angiotensin II concentrations are also increased in heart failure. The contribution of the renin-angiotensin system to peripheral vasoconstriction is further evident from the fact that angiotensin II antagonists such as saralasin, angiotensin II subtype 1 receptor, antagonists such as losartan, and angiotensin converting enzyme (ACE) inhibitors decrease systemic vascular resistance in heart failure. ${ }^{58}$ Several mechanisms appear to be involved in the increased renal release of renin into the circulation in heart failure. Increased renal sympathetic activity, decreased distal tubular sodium delivery, reduced renal perfusion pressure, and diuretic treatment may all contribute to increased plasma renin activity and increased angiotensin II levels in heart failure. Increased renal sympathetic activity is a reflection of the increase in systemic adrenergic activity that occurs in heart failure. A decreased distal tubular sodium load results from avid proximal reabsorption of sodium in heart failure. The macula densa cells respond to a decrease in distal tubular sodium chloride transport by increased renin release. Severe heart failure may be associated with not only decreased cardiac output but also relative hypotension, which may reduce renal perfusion pressure and subsequently be sensed by intrarenal baroreceptors which induce renin secretion. Diuretic treatment is also an important exogenous stimulus for renin release. In patients 
with heart failure receiving chronic diuretic treatment alone, plasma renin activity, angiotensin II levels, and aldosterone levels tend to increase. Indeed, in the absence of diuretic treatment in patients with heart failure, plasma renin activity and angiotensin II levels may only be minimally abnormal. The other neurohormonal abnormalities that are associated with heart failure may also contribute to the changes in plasma renin activity and circulating levels of angiotensin II in heart failure. Increased prostaglandins $\left(\mathrm{PGE}_{2}\right.$ and $\mathrm{PGI}_{2}$ levels) stimulate renin release, whereas increased concentrations of vasopressin and atrial natriuretic peptides may inhibit renin secretion. Endothelin, a vasoconstrictor peptide which is also raised in patients with heart failure, may stimulate renin and aldosterone release and enhance the conversion of angiotensin I to angiotensin II.

Activation of the circulating renin-angiotensin system in conjunction with enhanced sympathetic activity may produce deleterious effects on central and peripheral haemodynamics in heart failure. Increased systemic vascular resistance resulting from arteriolar vasoconstriction augments left ventricular ejection impedance, which is associated with decreased forward stroke volume and increased end systolic and end diastolic volumes. Without intrinsic cardiac and extrinsic peripheral compensatory changes, or with the failure of compensatory mechanisms, a progressive deterioration of central and peripheral haemodynamics and cardiac dynamics is likely to occur. In patients with overt heart failure, arterial distensibility is decreased and the normal left ventricular aortic coupling is disturbed. ${ }^{6}$ The increased circulating reninangiotensin-aldosterone axis may contribute to this abnormal arterial compliance, and ventriculo-aortic coupling may improve when treated with ACE inhibitors. As well as causing impairment of systolic function, activation of the renin-angiotensin system may also contribute to abnormal diastolic function, which often coexists along with systolic dysfunction in heart failure. ${ }^{5}$ Increased renin-angiotensinaldosterone and adrenergic activity contribute to venoconstriction, increased intracardiac volume and intracardiac pressures. Altered ventricular geometry and volume, along with increased left ventricular filling pressures, impair diastolic filling. The contribution of the renin-angiotensin system to vasoconstriction is further evident from the fact that the use of angiotensin II antagonists, such as saralasin, is associated with decreased systemic and pulmonary vascular resistance and increased cardiac output. ACE inhibitors, which also attenuate the vasoconstrictive effects of angiotensin II, have been shown to decrease systemic vascular resistance, arterial pressure, and ventricular filling pressures, with an increase in cardiac output in heart failure. More recently, the specific antagonists of angiotensin II subtype 1 receptors have been shown to cause peripheral vasodilatation, reduction in blood pressure, decreases in ejection impedance, and increases in cardiac out- put in heart failure. Thus it is apparent that the circulating renin-angiotensin system is activated in established heart failure, and one of the reasons for the use of inhibitors of the renin-angiotensin system in heart failure is to attenuate the deleterious effects of angiotensin II on cardiac and peripheral vascular dynamics.

The activated renin-angiotensin system may also contribute to abnormal coronary haemodynamics and myocardial energetics, which are common in patients with overt heart failure resulting from ischaemic or non-ischaemic dilated cardiomyopathy. Resting global coronary blood flow in patients with both ischaemic and non-ischaemic dilated cardiomyopathy and clinical heart failure is usually increased compared to that in patients without heart failure. Although global coronary blood flow is increased, coronary blood flow per $100 \mathrm{~g}$ of left ventricular mass may remain unchanged or even be less than normal. ${ }^{9}$ Myocardial oxygen consumption is also increased, and an increase in global coronary blood flow may reflect an increase in myocardial oxygen demand. The rate-pressure product-an index of myocardial oxygen demand-is, however, similar in patients with and without heart failure. Increased left ventricular wall stress is probably the major determinant for the increased myocardial consumption in patients with dilated cardiomyopathy. Coronary sinus venous oxygen content may decrease significantly in severe heart failure. This suggests that an augmented coronary blood flow may not be sufficient to meet the increase in metabolic demand. ${ }^{10}$ The activated renin-angiotensin system may contribute to the increased myocardial oxygen demand in heart failure. Altered peripheral vascular and cardiac haemodynamics with increased left ventricular wall stress are likely to be partly mediated by the activated reninangiotensin system. Furthermore, angiotensin II has been known to cause a dose dependent increase in myocardial contractility, which is associated with increased myocardial oxygen consumption. ${ }^{5}$

Coronary blood flow reserve appears to be diminished in patients with systolic ventricular failure resulting from ischaemic or nonischaemic dilated cardiomyopathy. The ratio of the maximum coronary blood flow after intracoronary injection of papaverine, dipyridamole, or adenosine, to resting flow-an index of coronary flow reserve-is substantially reduced in patients with dilated cardiomyopathy, compared to normal subjects. ${ }^{112}$ The mechanisms of impaired coronary flow reserve are multifactorial. Even in patients without atherosclerotic coronary artery disease, flow response to intracoronary infusion of acetylcholine is markedly blunted but response to adenosine remains relatively preserved, suggesting disturbed endothelium dependent coronary vasodilatation. ${ }^{13}$ In patients with obstructive atherosclerotic coronary artery disease, the most frequent cause of chronic congestive heart failure, impaired relaxation of the conductance vessels due to 
endothelial dysfunction, contributes to diminished coronary flow reserve. The contribution of angiotensin II to enhanced coronary vascular tone is suggested by the fact that angiotensin II subtype 1 receptor antagonists decrease coronary vascular tone in basal conditions, and it is likely that increased coronary vascular resistance and abnormal coronary flow reserve in heart failure may be partly mediated by the activated renin-angiotensin system. ${ }^{14}$ Thus one of the potential rationales for the use of the inhibitors of the renin-angiotensin system is to improve coronary haemodynamics and myocardial energetics in heart failure.

Activation of circulating neurohormones, including renin and angiotensin II as well as noradrenaline and atrial natriuretic peptides, has been shown in patients with overt heart failure and has been related to the severity of heart failure and prognosis. These circulating neurohormones are often near normal in many patients in a stable compensated clinical state. ${ }^{5}$ In the SOLVD trial, in patients with asymptomatic left ventricular dysfunction the circulating renin-angiotensin system activity was normal or near normal in the majority of patients. ${ }^{15}$ Despite normal plasma renin activity in these patients, treatment with enalapril blunted the progression of disease and subsequent development of overt congestive symptoms. It appears unlikely that these benefits might be mediated by inhibition of the circulating renin-angiotensin system alone. Thus it has been hypothesised that the tissue reninangiotensin system is activated in the absence of overt heart failure, and inhibition of system is likely to be beneficial in preventing ventricular and vascular remodelling and progression of heart failure, and therefore in improving survival.

The existence of the tissue renin-angiotensin system has been amply demonstrated by enzymatic biochemical and molecular biological techniques. Renin enzymatic activity has been shown in isolated cardiac myocytes. Cardiac renin and angiotensin agent messenger RNA (mRNA) expressions have been identified in the heart. ${ }^{5}$ The presence of ACE in cardiac tissues has also been recognised. In human myocardial tissues specific angiotensin receptors have also been identified. ${ }^{5}$ In experimental heart failure in animal models, multiple components of the renin-angiotensin system are locally activated. The expression of angiotensinogen has been documented in early heart failure in rats after experimental myocardial infarction. The increased expression of angiotensinogen has also been documented in tachycardia induced heart failure in animals. ${ }^{5}$ The expression of the angiotensin II subtype 1 receptor is also increased in both the right and left ventricular tissues within a few days after experimental myocardial infarction in animals. ${ }^{5}$ The mechanisms responsible for the induction of cardiac angiotensinogen, ACE inhibitor, and angiotensin receptor gene expression are not clearly understood, although increased wall stress or inflammatory cell or fibroblast migration into the healing myocardium may contribute to the increased expression of the cardiac renin-angiotensin system.

As well as increasing the expression of the renin-angiotensin system in various tissues of the myocardium, heart failure appears to stimulate the activity of the intrarenal angiotensin systems. ${ }^{5}$ The chronic compensated state of heart failure after experimental myocardial infarction in animals is associated with increased renal angiotensinogen mRNA levels. In experimental heart failure in animals, this increase in the intrarenal angiotensin system occurs without the concomitant increase in liver angiotensinogen mRNA levels. There is also no significant increase in kidney renin content or renal renin mRNA levels. Circulating angiotensinogen concentration, plasma renin concentration, and serum or renal ACE activities are also not increased, although intrarenal angiotensinogen expression is increased. The mechanism for the increased renal angiotensinogen mRNA levels in experimental heart failure remains unclear. Enhanced renal sympathetic activity and alterations in haemodynamics, such as low cardiac output or reduction in renal perfusion pressure, may contribute to this enhanced intrarenal expression of the renin-angiotensin system. Whatever the mechanisms might be, these findings suggest that the circulating renin-angiotensin system may not be activated in the compensated state of heart failure. In the studies in experimental animals with compensated heart failure, it has also been shown that chronic inhibition of ACE with relatively long acting ACE inhibitors such as enalapril can normalise increased renal angiotensinogen expression. It is likely that with normalisation of angiotensinogen expression, intrarenal angiotensin II synthesis is also decreased, and this may be associated with significant renal vasodilatation and natriuresis. ${ }^{5}$

ACE is found in high concentrations in the endothelium of all blood vessels, including aorta, large and small arteries, and the microvasculature. In the microvasculature, angiotensin I can be converted to angiotensin II in the presence of ACE, and angiotensin II can then diffuse into the myocardium. ${ }^{5}$

Although increased expression of the reninangiotensin system has been well documented in heart failure in experimental animals, the existence and changes in the expression of the tissue renin-angiotensin system and its potential effects on cardiovascular dynamics have not been adequately assessed up to now in patients with heart failure. Some preliminary studies, however, have suggested the existence of tissue renin-angiotensin system in explanted human myocardial tissue. ${ }^{16}$ It has been shown that angiotensin II exerts a positive inotropic effect on isolated human atrial and ventricular trabecular muscles. In failing human atrial tissue, there is a dose dependent positive inotropic effect of both angiotensin I and angiotensin II, and the positive inotropic effect of angiotensin II can be blocked with treatment with enalaprilat, which suggests a potential role of cardiac tissue ACE. Intracoronary infusion of enalaprilat, which does not pro- 
duce significant systemic haemodynamic changes, is associated with significant impairment of systolic and contractile function of the left ventricle, suggesting the existence of a tissue renin-angiotensin system in patients with idiopathic dilated cardiomyopathy. ${ }^{17}$ Along with a decrease in contractile function, there was a decrease in calculated coronary vascular resistance following intracoronary infusion of enalaprilat in these patients. These findings suggest that in patients with idiopathic dilated cardiomyopathy, tissue angiotensin II enhances the myocardial inotropic state as well as contributing to potential coronary vasoconstriction. In the human heart, nonACE biochemical pathways for conversion of angiotensin I to angiotensin II (chymase) have also been identified. ${ }^{18}$ The relative importance of ACE and chymase in human hearts, however, remains unclear. It is possible that human cardiac tissue angiotensin II generation might still occur, despite systemic inhibition of ACE, because of the presence of chymase.
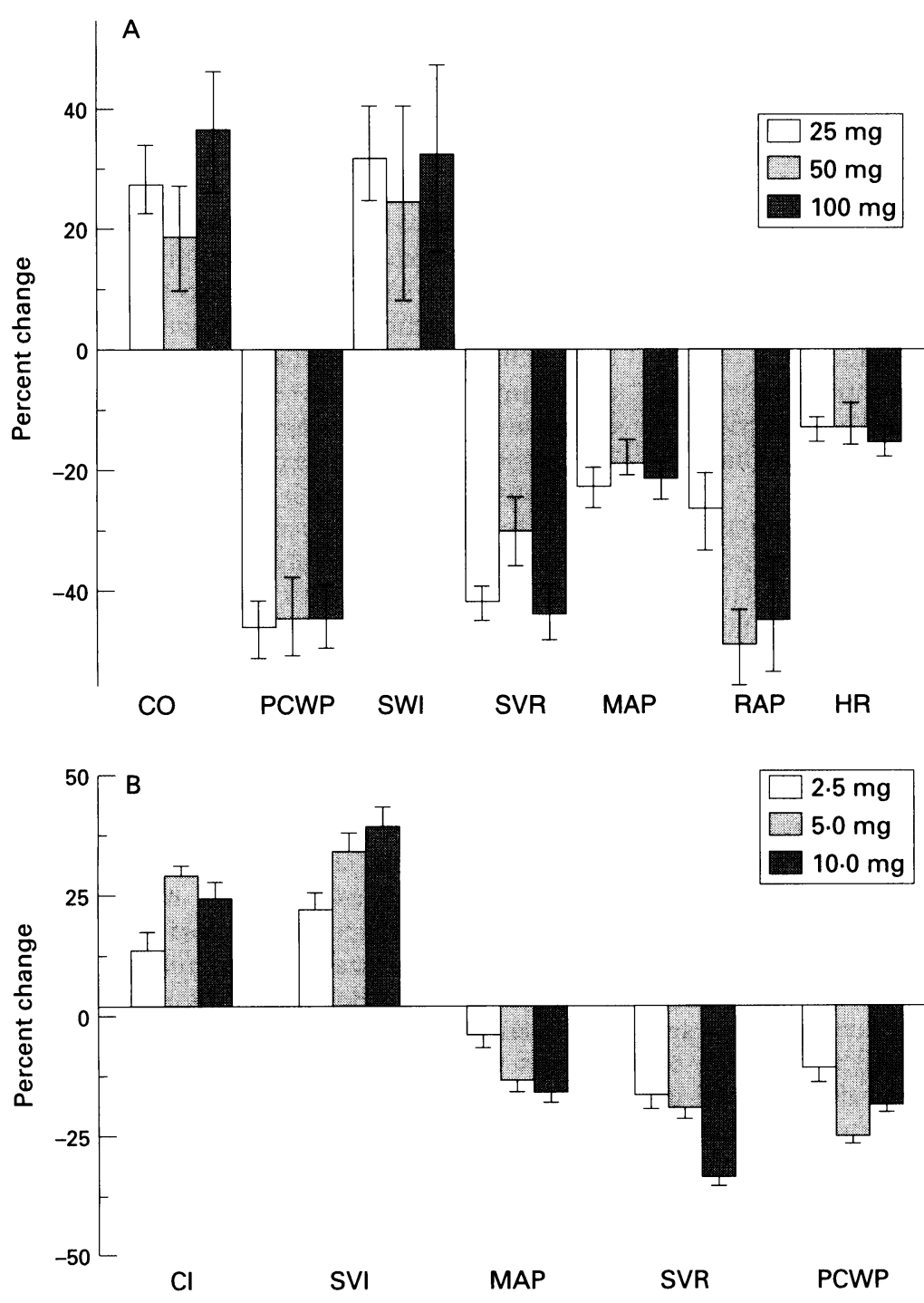
Figure 1(A) Changes in cardiac output (CO), pulmonary capillary wedge pressure
(PCWP), stroke work index (SWI), systemic vascular resistance (SVR), mean arterial (PCWP), stroke work index (SWI), systemic vascular resistance (SVR), mean arterial
pressure (MAP), right atrial pressure $(R A P)$, and heart rate (HR) after 25,50 , and 100 $m g$ of oral captopril $(n=10)$. Published with permission from Ader et al. ${ }^{21}$ (B) Changes in cardiac index (CI), stroke volume index (SVI), MAP, SVR, and mean PCWP after $2 \cdot 5,5 \cdot 0$, and $10 \mathrm{mg}$ of oral enalapril $(n=12)$. Published with permission from DiCarlo et al. ${ }^{22}$
The relevance of the existence of the tissue renin-angiotensin system, particularly in the heart, vascular bed, and kidney, is that it may play a role in the pathophysiology of heart failure. Activation of the tissue renin-angiotensin system in the vascular bed may not only contribute to maintaining high vascular tone in heart failure, but may also contribute to smooth muscle cell hypertrophy and vascular remodelling. Coronary haemodynamic and myocardial energetics may also be adversely affected by enhanced vascular tissue reninangiotensin system activity. Not only may myocardial oxygen demand be increased, but myocardial perfusion may also be impaired, inducing an imbalance between supply and demand and contributing to myocardial ischaemia, which in turn may contribute to myocyte loss. Enhanced activity of the cardiac renin-angiotensin system may also play a significant role in ventricular remodelling in addition to inducing abnormalities of systolic and diastolic function. The growth promoting effects of angiotensin II may be contributory to maladaptive myocyte hypertrophy and architectural derangements of the extracellular matrix. One of the potential benefits of the inhibition of the tissue renin-angiotensin system is the attenuation of this vascular and cardiac remodelling, which has been suggested as a fundamental mechanism for progression of heart failure. It has been reported that tissue ACE could be inhibited in humans with the use of orally administered ACE inhibitors, and that significant inhibition of ACE activities in the kidney, heart, and aortic and venous tissues occurs after short term administration of oral ACE inhibitors. ${ }^{19}$ In patients with established heart failure, long term treatment with ACE inhibitors can also attenuate ventricular remodelling. ${ }^{20}$

\section{Systemic and regional haemodynamic effects of inhibition of the renin- angiotensin system}

Many studies have been performed to assess acute and chronic haemodynamic effects of various $A C E$ inhibitors with different pharmacokinetic properties. ${ }^{4}$ Qualitatively, the systemic haemodynamic effects of ACE inhibitors are similar, irrespective of the differences in pharmacokinetic properties. Captopril is a sulphydryl containing, relatively short acting ACE inhibitor. Enalapril, on the other hand, is a longer acting non-sulphydryl prodrug and requires in vivo hepatic esterolysis to the active metabolite, enalaprilat. The acute systemic haemodynamic responses to captopril and enalapril are illustrated in fig $1 \mathrm{~A}$ and B. ${ }^{2122}$ The haemodynamic effects are characterised by a significant reduction in right atrial and pulmonary capillary wedge pressure, along with a reduction in pulmonary artery and systemic arterial pressures. Systemic vascular resistance consistently declines, and, in general, pulmonary vascular resistance is also decreased. Cardiac output and stroke volume tend to increase, and heart rate either decreases slightly or remains unchanged. It is 
apparent that the haemodynamic effects of captopril and enalapril are similar. Lisinopril, quinapril, ramipril, cilazapril, zofenopril, perindopril, fosinopril, and benazepril are other long acting ACE inhibitors which also produce similar systemic haemodynamic effects in patients with chronic heart failure. The beneficial changes in systemic haemodynamics in response to ACE inhibitors have also been determined during exercise. When assessed at comparable workloads, the magnitude of reduction in systemic vascular resistance and increase in cardiac output is greater following ACE inhibitor therapy. There is also a decrease in pulmonary capillary wedge pressure along with an increase in the stroke volume during exercise. The beneficial haemodynamic effects of ACE inhibitors in patients with chronic heart failure appear to persist during long term treatment in the majority of patients..$^{42122}$ A sustained reduction in pulmonary capillary wedge pressure and right atrial pressure, and increases in cardiac output and stroke volume, have been shown. However, in approximately $27 \%$ of patients, haemodynamic improvement initially observed may not persist during chronic treatment with ACE inhibitors. It is of interest that in approximately $20 \%$ of patients there was no initial haemodynamic effect, but in several of these patients haemodynamics improved during continued treatment. ${ }^{23}$

The mechanism of improvement in systemic haemodynamics and left ventricular performance are likely to be related to improvement in both systolic and diastolic function of the left ventricle. ${ }^{6}$ The reduction in systemic vascular resistance, ejection impedance, and left ventricular afterload causes an increase in left ventricular ejection fraction and forward stroke volume, and a reduction in left ventricular end systolic and end diastolic volume along with a decrease in left ventricular diastolic pressure and pulmonary venous pressure. An improvement in ventriculo-aortic coupling following ACE inhibitor treatment also assists left ventricular pump function. Improved diastolic filling characteristics of the left ventricle may contribute in improving haemodynamics as well.

A concomitant improvement in right ventricular systolic and diastolic function contributes to improved forward stroke volume and cardiac output following ACE inhibitor treatment. The changes in contractile state with the use of ACE inhibitors are likely to be variable. Intracoronary infusion of $\mathrm{ACE}$ inhibitors, which does not usually produce significant systemic effects, has been shown to decrease contractile state because of inhibition of angiotensin II mediated positive inotropic effects. ${ }^{17}$ Thus there is potential for decreased contractility during acute inhibition of the effect of angiotensin II. However, in experimental animals chronic inhibition of angiotensin II with ACE inhibitors has been shown to improve myocardial contractile performance. Upregulation of the myocardial $\beta$ adrenergic receptors, due to chronic sympatho-inhibition and a consequent increase in myocardial contractile response to catecholamines, is a possible mechanism. ${ }^{24}$ In cardiomyopathic hamsters, prevention of cardiac myosin phenotype alterations by ACE inhibitors has been suggested as another mechanism of improved contractility. However, it remains to be determined whether or not chronic inhibition of the effects of angiotensin II by ACE inhibitors improves overall contractile performance in patients with chronic heart failure due to ischaemic or nonischaemic dilated cardiomyopathy.

In patients with established heart failure, chronic inhibition of the renin-angiotensin system with ACE inhibitors has been shown to decrease left ventricular mass and left ventricular wall stress, which may contribute to improved ventricular performance. ${ }^{20} \mathrm{ACE}$ inhibitors also have the potential to improve coronary haemodynamics and myocardial energetics and, secondarily, to improve cardiac performance. ${ }^{25}$

ACE inhibitors produce peripheral vasodilatation by various mechanisms. Direct inhibition of the vasoconstrictive effects of angiotensin II produces peripheral vasodilatation. However, increased bradykinin levels due to inhibition of the enzyme kininase II (converting enzyme), which is necessary for the breakdown of bradykinin to inactive bradykinin peptides, may be relevant in the peripheral vasodilatation produced by ACE inhibitors. Furthermore, increased bradykinin may also cause enhanced release of prostacyclin, which is also a potent vasodilator. However, inhibition of the vasoconstrictive effect of angiotensin II appears to be an important mechanism, as angiotensin II receptor subtype 1 antagonist tends to induce similar peripheral vasodilatation. ACE inhibitors can improve renal haemodynamics in patients with heart failure. Angiotensin II is a potent renal vasoconstrictor, predominantly affecting efferent arterioles. In the absence of significant hypotension in response to ACE inhibitors, renal blood flow may increase significantly. Glomerular filtration rate, however, may not change. Many studies have shown an increase or no change or even a decrease in glomerular filtration rate. ${ }^{6}$ During long term ACE inhibitor treatment, renal function in some patients improved in association with an increase in free water clearance as well as an increase in sodium excretion. However, in many patients-particularly with severe heart failure-ACE inhibitor treatment may be associated with an increase in BUN and creatinine. ${ }^{6}$

ACE inhibitors may also produce favourable effects on coronary haemodynamics and myocardial energetics in patients with chronic congestive heart failure resulting from left ventricular systolic dysfunction with or without associated coronary artery disease. ACE inhibitors consistently decrease myocardial oxygen demand, and myocardial oxygen consumption declines in parallel. This decreased myocardial oxygen demand and consumption results primarily from a decrease in the heart rate-blood pressure product. 
Figure 2 Intracoronary infusions of enalaprilat in dogs increase the cross sectional areas of the epicardial coronary arteries (intravascular imaging), suggesting dilatation of the average peak velocity (intravascular Doppler) also increases, suggesting a vasodilator effect of enalaprilat on the coronary resistance vessels

${ }^{\star}$ denotes significance).

Published with permission from Sudhir et al. ${ }^{14}$
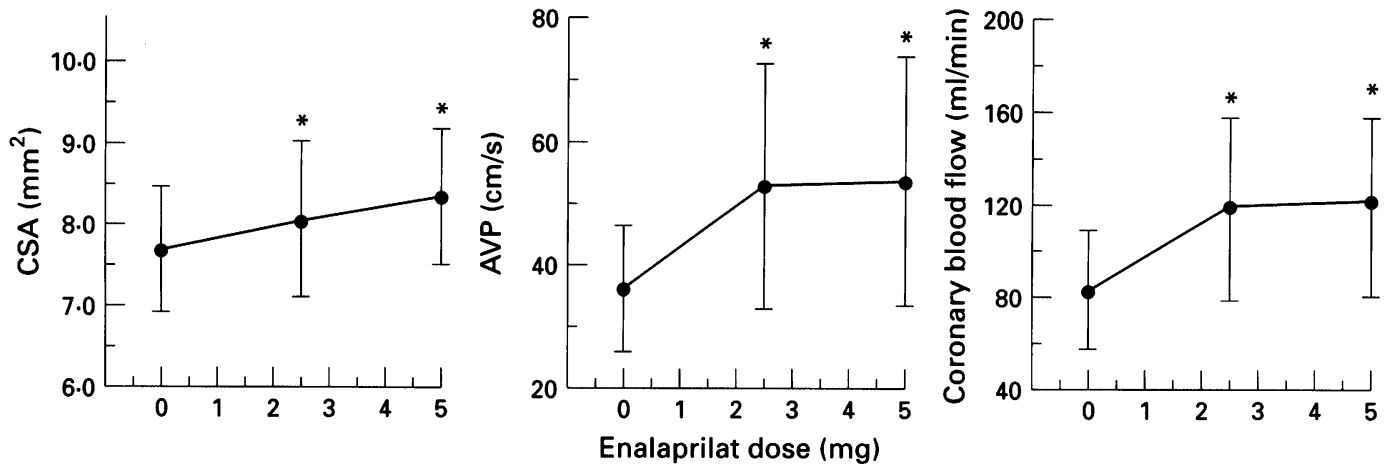

However, a reduction in left ventricular wall stress may also be contributory. ACE inhibitors also have the potential to increase coronary blood flow by primary reduction in coronary vascular resistance. ACE inhibitors produce vasodilatation of both conductance and resistance vessels (fig 2). ${ }^{14}$ Vasodilatation of the conductance vessels is partly mediated by endothelium dependent mechanisms, as concurrent administration of a nitric oxide synthase inhibitor partly attenuates vasodilatation of the epicardial coronary arteries. Dilatation of the resistance vessels with ACE inhibitors is partly mediated by bradykinin, since bradykinin antagonists attenuate the magnitude of vasodilatation of the resistance vessels. Whatever the mechanisms of vasodilatation of coronary vascular bed, ACE inhibitors have the potential to increase coronary blood flow in excess of myocardial oxygen demand. ${ }^{26}$ These favourable effects of ACE inhibitors on coronary haemodynamics may relieve myocardial ischaemia in some patients with heart failure due to dilated cardiomyopathy.

ACE inhibitors appear to possess both arteriolar and venodilating properties. In patients with heart failure, there is usually a substantial decrease in forearm vascular resistance and increase in forearm blood flow, and also an increase in peripheral venous capacitance in response to ACE inhibitors. Following chronic treatment with an ACE inhibitor, skeletal muscle blood flow may also increase. This peripheral haemodynamic effect of ACE inhibitors may contribute to improved central haemodynamics and exercise tolerance in patients with chronic heart failure treated with ACE inhibitors. ${ }^{4}$

\section{Clinical efficacy of inhibition of the renin-angiotensin system in established heart failure}

Improvement in symptoms of congestive heart failure and exercise tolerance with ACE inhibitors have been documented in a number of randomised prospective placebo controlled studies. ${ }^{4}$ The Captopril Multicenter Research Group reported that after three months of treatment with captopril, the NYHA functional class improved in $61 \%$ of patients treated with captopril, whereas only $24 \%$ of placebo treated patients improved. ${ }^{27}$ Treadmill exercise time increased significantly more in the captopril treatment group than in the placebo group. Treatment with enalapril has also been reported to improve clinical class and exercise tolerance in patients with congestive heart failure; both improvement in functional class and exercise duration have been Chalmers et al reported that lisinopril treatment improved exercise duration during a 12 week placebo controlled trial. ${ }^{28}$ Similarly, treatment with quinapril is also associated with improved exercise tolerance in patients with congestive heart failure, compared to placebo. ${ }^{29}$ It should be noted, however, that improvement in exercise duration is not uniformly observed in patients treated with ACE inhibitors.

In a few studies, the relative efficacy of the various ACE inhibitors has been compared in prospective controlled randomised studies. Comparable clinical improvement was observed between captopril and enalapril, captopril and lisinopril, and lisinopril and enalapril. ${ }^{4}$ The results of these studies suggest that improvement in clinical class and exercise tolerance are similar, irrespective of the pharmacokinetic and pharmacodynamic differences between various ACE inhibitors.

The relative efficacy of ACE inhibitors and of digoxin has also been evaluated in prospective controlled studies. ${ }^{3031}$ In some studies, ACE inhibitors were found to be more effective than digoxin in improving clinical status and exercise tolerance. In other studies, however, no significant differences between digoxin and ACE inhibitors were observed in improving clinical status and exercise tolerance. In the VA Heart Failure Trial II, the clinical efficacy and mortality rate were compared between hydralazine, isosorbide dinitrate, and enalapril. ${ }^{32}$ Although with enalapril there was a greater survival benefit, the exercise tolerance and improvement in clinical class and increase in maximum oxygen consumption during exercise were greater with hydralazine and isosorbide dinitrate than with enalapril. The clinical efficacy and neuroendocrine changes with the angiotensin II subtype 1 receptor antagonist, losartan, have been compared to those with enalapril in patients with moderate or severe chronic heart failure. ${ }^{33}$ No significant differences were observed between losartan and enalapril in improving exercise capacity or clinical status. The changes in neurohumoral profile-that is, in observed in patients treated with enalapril. 


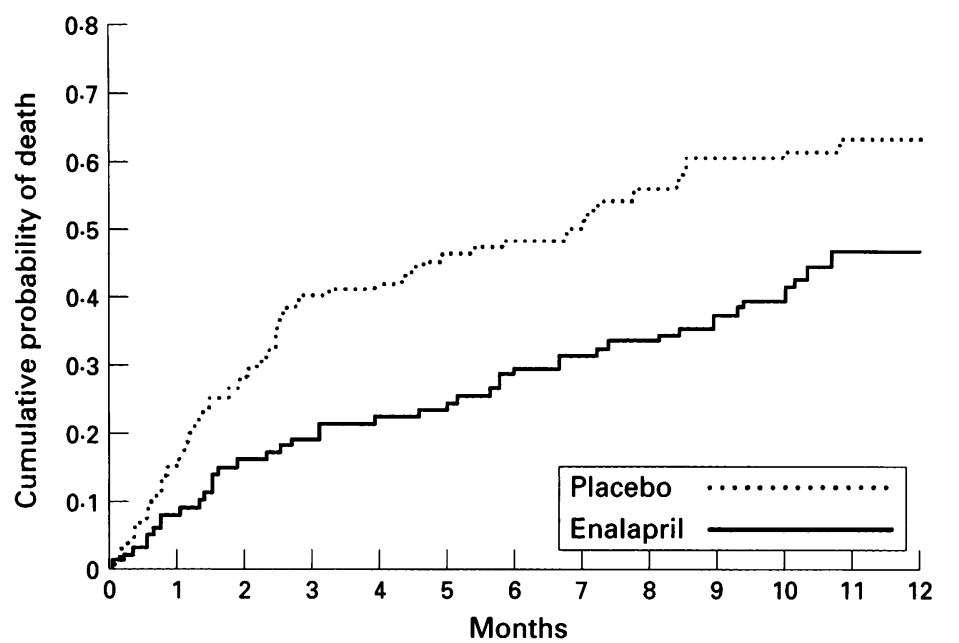

$\begin{array}{llllllllllllll}\text { Placebo n: } & 128 & 102 & 78 & 63 & 59 & 83 & 47 & 42 & 34 & 30 & 24 & 16 & 17\end{array}$

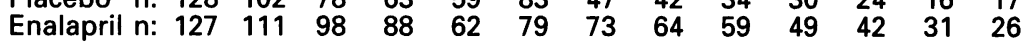

Figure 3 The effect of enalapril compared to placebo on mortality in patients with severe congestive heart failure. Reproduced with permission from the CONSENSUS Trial Study Group. ${ }^{34}$ of the renin-angiotensin system by enalapril on mortality and on non-fatal events such as hospital admission in patients with decreased left ventricular ejection fraction (less than 36\%) and with established mild to moderate congestive heart failure. In the treatment arm of this trial, patients had symptoms of mild congestive heart failure, and most were in NYHA functional class I or II. Many patients in this study were receiving digoxin, glycosides, and no diuretics, and in the treatment arm enalapril was added $(2 \cdot 5-20 \mathrm{mg}$ daily, fig 4$)$. At the end of two years, mortality in the enalapril treated group was approximately $20 \%$ and in the placebo group approximately $25 \% .{ }^{35}$ Thus enalapril reduced the risk of death by $23 \%$. At the end of 3.5 years, the mortality risk reduction with enalapril was about $16 \%$. In addition, enalapril resulted in a $30 \%$ reduction in the risk of hospital admission for heart failure for up to two years of treatment.

In the VA Heart Failure Trial II, the effect of enalapril was compared to the combination of hydralazine and isosorbide dinitrate in patients with mild congestive heart failure (fig 5). ${ }^{32}$ In the VA Heart Failure Trial I, impact of treatment with hydralazine/isosorbide dinitrate on mortality compared to conventional treatment-that is, digoxin and diuretics-was assessed in patients with similar severity of heart failure. In this trial, treatment with hydralazine and isosorbide dinitrate was associated with risk of reduction in mortality by $38 \%, 25 \%$, and $23 \%$, after one, two, and three years of follow up. ${ }^{36}$ In the VA Heart Failure Trial II, the magnitude of reduction in the risk of mortality with enalapril was greater than that with hydralazine and isosorbide dinitrate treatment (fig 5). During the course of the trial, mortality was $32.8 \%$ in patients randomised to enalapril, and $38.2 \%$ in those randomised to hydralazine and isosorbide dinitrate. The lower mortality rate in the enalapril group was primarily due to a decreased incidence of sudden death. There was no significant difference in mortality from progressive congestive heart failure between the two treatment groups. A meta-analysis of several smaller studies has indicated that ACE inhibitors can significantly reduce the risk of mortality in patients with heart failure. ${ }^{37}$ In

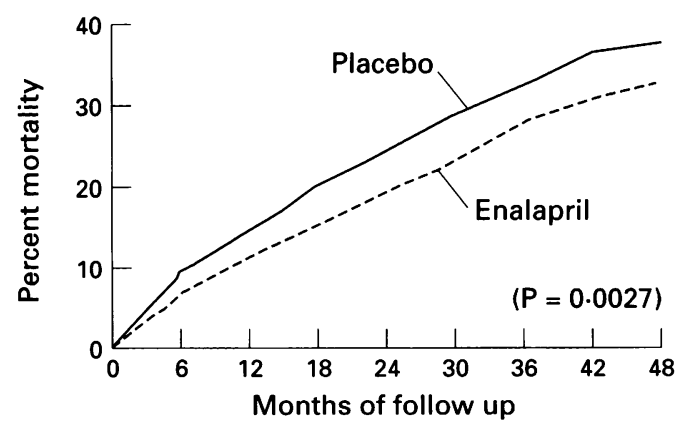

Figure 4 The effect of enalapril compared to placebo on mortality in patients with mild to moderately severe congestive heart failure. Reproduced with permission from the SOLVD Investigators. ${ }^{35}$ 
Figure 5 The effect of enalapril compared to hydralazine and isosorbide dinitrate on mortality in patients with mild to moderate congestive heart failure. Reproduced with permission from Cohn

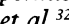

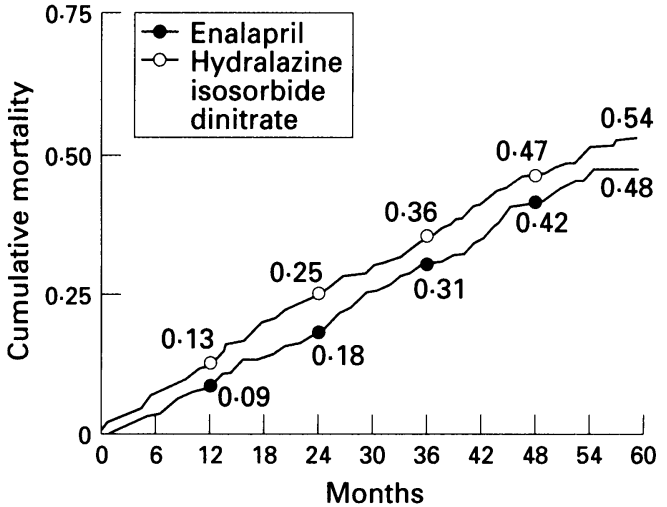

patients with established heart failure following acute myocardial infarction, ACE inhibitors have been shown to decrease mortality. The Acute Infarction Ramipril Efficacy (AIRE) study enrolled and randomised patients with acute myocardial infarction who had evidence of congestive heart failure between day 3 and day 10 after acute myocardial infarction. ${ }^{38}$ There was a $27 \%$ reduction in risk of mortality with treatment with ramipril in this study. Thus these studies suggest that in patients with established heart failurewhether acute or chronic-treatment with ACE inhibitors can improve survival. Furthermore, inhibition of the renin-angiotensin system in patients with established heart failure is associated with improved clinical and haemodynamic status, exercise performance, and quality of life. Furthermore, treatment with ACE inhibitors in patients with established coronary artery disease appears to be associated with decreased incidence of unstable angina and re-infarction. In established heart failure, therefore, inhibition of the reninangiotensin system should be considered a standard and essential treatment.

1 Garg R, Packer M, Pitt B, Yusuf S. Heart failure in the 1990s: evolution of a major public health problem in cardiovascular medicine. 7 Am Coll Cardiol 1993;22:3-5A

2 Parameshwar J, Shackell MM, Richardson A, Poole-Wilson PA, Sutton GC. Prevalence of heart failure in three genral practices in northwest London. $\mathrm{Br} f \mathrm{Gen}$ Pract 1992;42:287-9.

3 McCall D. Epidemiology, etiology and natural history. In McCall D, Rahimtoola SH, eds. Heart failure. New York: Chapman and Hall, 1995:1-13.

4 Creager MA, Cusco JA. Treatment of congestive heart failure with angiotensin converting enzyme inhibitors. In McCall D, Rahimtoola SH, eds. Heart failure. New York: Chapman and Hall, 1995:316-44.

5 Hirsch AT, Mullerlerleile M, Dzau VJ. Cardiovascular tissue angiotensin systems: Activation and actions in heart failangiotensin systems: Activation and actions in heart failure. In: McCall D, Rahimtoola SH, eds. Hean

6 Chatterjee K. Systolic ventricular failure. In: Parmley WW, Chatterjee K, eds. Cardiology. Philadelphia: JB Lippincott, 1987:1-44.

7 Merrill AJ, Morrison JL, Brannon ES. Concentration of renin in renal venous blood in patients with chronic heart failure. $\mathrm{Am} \mathcal{F}$ Med 1946;1:468-72.

8 Crozier I, Ikram H, Awan N, Cleland J, Stephen N, Dickstein $\mathrm{K}$, et al. Losartan Hemodynamic Study Group. Losartan in heart failure. Hemodynamic effects and tolerability. Circulation 1995;91:691-7.

9 De Marco T, Chatterjee K, Rouleau JL, Parmley WW. Abnormal coronary hemodynamics and myocardial en getics in patients with chronic heart failure caused by chemic heart disease and dilated cardiomyopathy. $A m$ Heart 7 1988;115:809-15.

10 White M, Rouleau JL, Ruddy TD, De Marco T, Moher D, Chatteriee K. Decreased coronary sinus oxygen content: predictor of adverse prognosis in patients with severe congestive heart failure. F Am Coll Cardiol 1991;18: $1631-7$

11 Inoue T, Sakai Y, Morooka S, Hayashi T, Takayanagi K, Yamanaka $\mathrm{T}$, et al. Coronary flow reserve in patients with dilated cardiomyopathy. Am Heart $\mathcal{f} 1993 ; 125: 93-8$.

12 Neglia D, Parodi O, Gallopin M, Sambuceti G, Giorgetti A, Pratali L, et al. Myocardial blood flow response to pacing tachycardia and to dipyridamole infusion in patients with dilated cardiomyopathy without overt heart failure. A quantitative assessment by positron emission tomography. Circulation 1995;92:796-804

13 Treasure CB, Vita JA, Cox DA, Fish RD, Gordon JB, Mudge GH, et al. Endothelium-dependent dilation of the coronary microvasculature is impaired in dilated cardiomyopathy. Circulation 1990;81:772-9.

4 Sudhir K, MacGregor JS, Gupta M, Barbant SD, Redberg $\mathrm{R}$, Yock PG, et al. Effect of selective angiotensin II receptor antagonism and angiotensin converting enzyme inhibition on the coronary vasculature in vivo. Intravascular two-dimensional and Doppler ultrasound studies. Circulation 1993;87:931-8.

15 The SOLVD Investigators. Effect of enalapril on mortality and the development of heart failure in asymptomatic patients with reduced left ventricular ejection fractions (published erratum appears in N Engl f Med 1992;327: 1768). $N$ Engl f Med 1992;327:685-91.

16 Moravec CS, Schluchter MD, Paranandi L, Czerska B, Stewart RW, Rosenkranz E, et al. Inotropic effects of angiotensin II on human cardiac muscle in vitro. Circulation 1990;82:1973-84.

17 Foult JM, Tavolaro O, Antony I, Nitenberg A. Direct myocardial and coronary effects of enalaprilat in patients with dilated cardiomyopathy: assessment by a bilateral $337-44$.

18 Urata H, Kinoshita A, Misono KS, Bumpus FM, Husain A. Identification of a highly specific chymase as the major angiotensin II-forming enzyme in the human heart (published erratum appears in $\mathcal{F}$ Biol Chem 1991;266:12114). $f$ Biol Chem 1990;265:22348-57.

9 Erman A, Winkler J, Chen-Gal B, Rabinov M, Zelykovski $A$, Tadjer $\mathrm{S}$, et al. Inhibition of angiotensin converting enzyme by ramipril in serum and tissue of man. $\mathcal{F}$ Hypertens 1991;9:1057-62.

20 Doherty NE, Seelos KC, Suzuki J, Caputo GR, O'Sullivan M, Sobol SM, et al. Application of cine nuclear magnetic resonance imaging for sequential evaluation of response to angiotensin- converting enzyme inhibitor therapy in dilated cardiomyopathy. $7 \mathrm{Am}$ Coll Cardiol 1992;19: 1294-302.

21 Ader R, Chatterjee K, Ports T, Brundage B, Hiramatsu B, Parmley W. Immediate and sustained hemodynamic and clinical improvement in chronic heart failure by an oral angiotensin-converting enzyme inhibitor. Circulation 1980;61:931-7.

22 DiCarlo L, Chatteriee K, Parmley WW, Swedberg K, Atherton B, Curran D, et al. Enalapril: a new angiotensin-converting enzyme inhibitor in chronic heart fail-
ure: acute and chronic hemodynamic evaluations. $₹ \mathrm{Am}$ Coll Cardiol 1983;2:865-71.

23 Packer M, Medina N, Yushak M, Meller J. Hemodynamic patterns of response during long-term captopril therapy for severe chronic heart failure. Circulation 1983;68 803-12.

24 Maisel AS, Phillips C, Michel MC, Ziegler MG, Carter SM. Regulation of cardiac beta-adrenergic receptors by captopril. Implications for congestive heart failure. Circulation 1989;80:669-75.

25 Chatterjee K, Rouleau JL, Parmley WW. Haemodynamic and myocardial metabolic effects of captopril in chronic heart failure. Br Heart $\mathcal{1}$ 1982;47:233-8.

26 De Marco T, Daly PA, Liu M, Kayser S, Parmley WW, Chatterjee K. Enalaprilat, a new parenteral angiotensinconverting enzyme inhibitor: rapid changes in systemic and coronary hemodynamics and humoral profile in chronic heart failure. $\mathcal{F} \mathrm{Am}$ Coll Cardiol 1987;9:1 131-8.

27 Captopril Multicenter Research Group. A placebo-controlled trial of captopril in refractory chronic congestive heart failure. $7 \mathrm{Am}$ Coll Cardiol 1983;2:755-63.

28 Chalmers JP, West MJ, Cyran J, De La Torre D, Englert M, Kramar M, et al. Placebo-controlled study of lisinopril in congestive heart failure: a multicentre study. $\mathcal{f}$ pril in congestive heart failure: a multicentre
Cardiovasc Pharmacol 1987;9 suppl 3:S89-97.

29 Riegger GA. Effects of quinapril on exercise tolerance in patients with mild to moderate heart failure. Eur Heart $\mathcal{F}$ patients with mild

30 The Captopril-Digoxin Multicenter Research Group Comparative effects of therapy with captopril and Comparative effects of therapy with captopril and digoxin in patients with

31 Davies RF, Beanlands DS, Nadeau C, Phaneuf D, Morris A Arnold JM, et al. Canadian Enalapril Versus Digoxin Study Group. Enalapril versus digoxin in patients with congestive heart failure: a multicenter study. $\mathrm{F} \mathrm{Am} \mathrm{Coll}$ Cardiol 1991;18:1602-9.

32 Cohn JN, Johnson G, Ziesche S, Cobb F, Francis G, Tristani F, et al. A comparison of enalapril with hydralazine-isosorbide dinitrate in the treatment of chronic congestive heart failure. $N$ Engl F Med 1991;325 303-10.

33 Dickstein K, Chang P, Willenheimer R, Haunso S, Remes J, Hall C, et al. Comparison of the effects of losartan and enalapril on clinical status and exercise performance in patients with moderate or severe chronic heart failure. $\mathcal{F}$ Am Coll Cardiol 1995;26:438-45.

34 The CONSENSUS Trial Study Group. Effects of enalapril on mortality in severe congestive heart failure. Results of the Cooperative North Scandinavian Enalapril Survival 
Study (CONSENSUS). $N$ Engl $\mathcal{f}$ Med 1987;316: 1429-35.

35 The SOLVD Investigators. Effect of enalapril on survival in patients with reduced left ventricular ejection fractions and congestive heart failure. $N$ Engl f Med 1991;325: 293-302.

36 Cohn JN, Archibald DG, Ziesche S, Franciosa JA, Harston mortality in chronic congestive heart failure. Results of a
Veterans Administration Cooperative Study. $N$ Engl $\mathcal{F}$ Med 1986;314:1547-52.

37 Furberg CD, Yusuf S. Effect of drug therapy on survival in chronic congestive heart failure. Am $\mathcal{f}$ Cardiol 1988;62: 41-5A.

38 The Acute Infarction Ramipril Efficacy (AIRE) Study Investigators. Effect of ramipril on mortality and morbidity of survivors of acute myocardial infarction with clinical evidence of heart failure. Lancet 1993;342:821-8. 